\title{
CS Research Square \\ Are "fibrosis-like" strips on chest CT in patients with COVID-19 pneumonia true fibrosis?
}

Qiulian Sun

Xinjian Xu

Hai Yang

Guanliang Wang

Jingjing Li ( $\square$ lijjdoc@126.com )

Juan $\mathrm{Wu}$

\section{Research Article}

Keywords: COVID-19, Pneumonia, CT, "Fibrosis-like" strips

Posted Date: October 7th, 2020

DOI: https://doi.org/10.21203/rs.3.rs-84236/v2

License: (9) This work is licensed under a Creative Commons Attribution 4.0 International License.

Read Full License 


\section{Abstract}

Objective: To investigate the evolution and characteristics of "fibrosis-like" strips on chest CT in patients with COVID-19 pneumonia.

Methods: From January 17, 2020, to February 27, 2020, inpatients diagnosed with COVID-19 pneumonia in Enze Hospital, Taizhou, Zhejiang Province, were selected. The chest CT data of the patients were collected, and patients were included in the present study according to the predefined inclusion criteria. The dynamic evolution process and outcome of "fibrosis-like" strips on chest CT were analyzed.

Results: A total of 36 patients (20 males and 16 females) with COVID-19 were included in the study, all of whom were diagnosed with mild or common COVID-19; "fibrosis-like" strips were observed in 29 patients (80.6\%) on the first chest CT scan, and "fibrosis-like" strips were observed in 7 patients (19.4\%) on the second chest CT scan, Repeated chest CT during the course of treatment showed that all patients with "fibrosis-like" strips had varying degrees of absorption. "Fibrosis-like" strips in 15 patients demonstrated a trend of first increasing in number, and then decreasing in number; "fibrosis-like" strips in 3 patients demonstrated a trend of first decreasing in number first, followed by an increase in number and then a decrease in number; and "fibrosis-like" strips in 18 patients demonstrated a trend of a gradual decrease in number. Follow-up chest CT after treatment showed that "fibrosis-like" strips in 15 patients completely disappeared in both lungs, and "fibrosis-like" strips completely disappeared in unilateral lung of 8 cases.

Conclusion: In the present study, we observed that "fibrosis-like" strips in COVID-19 patients had characteristics of early appearance and rapid morphological changes as well as rapid absorption, suggesting that "fibrosis-like" strips may be a sign of subsegmental atelectasis rather than fibrotic changes.

\section{Introduction}

Since December 2019, COVID-19 has spread rapidly in more than 200 countries and territories worldwide. Globally, as of 6:18 pm CEST, July 22, 2020, there have been 14,765,256 confirmed cases of COVID-19, including 612,054 deaths, reported to the WHO ${ }^{[1]}$. The rapid spread of COVID-19 has become a serious threat to global public health security; therefore, fast identification, testing, and timely isolation and treatment of confirmed cases are critical for controlling the epidemic. Chest CT plays an important role in the diagnosis and treatment evaluation of COVID-19. The CT features of COVID-19 pneumonia mainly include different types of ground-glass opacities, consolidation, and "fibrosis-like" strips. Currently, the exact imaging and pathological mechanisms of "fibrosis-like" strips remain unclear. Most clinicians believe that they represent pulmonary fibrotic changes and consider that they may be a feature of the reversal of COVID-19 [2]. However, our experience shows that there may be another explanation for "fibrosis-like" strips. Therefore, we performed this study to investigate the evolving process and outcome of "fibrosis-like" strips on chest CT in COVID-19 patients who were admitted to our hospital. 


\section{Methods}

1. Study design and patient selection

This was a retrospective, observational study conducted at Taizhou Enze Hospital. A total of 86 patients with COVID-19 confirmed by real-time-polymerase chain reaction (RT-PCR) tests of sputum, throat swabs, lower respiratory tract secretion from January 17, 2020, to February 27, 2020 were selected. The inclusion criteria were as follows: 1 . the patient's first visit and subsequent clinical treatments as well as examinations were all in our hospital; 2. "fibrosis-like" strips were observed on chest CT during the clinical course; and 3 . the patient received at least 3 or more chest CT examinations. This study was approved by the Medical Ethics Committee of Taizhou Enze Hospital in Zhejiang Province.

\section{Imaging methods}

Chest CT examination was performed using high definition discovery CT750HD (GE Healthcare), with the patient in the prone position, with the head advanced, and with deep inhalation after holding the breath; The scanning parameters were as follows: pitch, 0.984 ; tube voltage, $120 \mathrm{kV}$; automatic tube current; layer thickness/layer interval, 5mm; reconstructed layer thickness/layer interval, $1 \mathrm{~mm}$; and scanning range from the apical lung to the bottom of the bilateral diaphragm. Reconstruction methods: curved reconstruction of the target bronchus was implemented.

\section{Image data collection}

The extracted data included the chest CT images of all COVID-19 patients admitted to our hospital at admission, during hospitalization, and after discharge.

\section{Results}

\section{General clinical information}

As of February 27, 2020, a total of 36 patients with COVID-19 pneumonia met the inclusion criteria and were included in the present study, all of whom had mild or common COVID-19. Twenty patients were men and sixteen patients were women, with a median age of 54 years (range 27-79 years). All patients had a travel history or residence history of Wuhan.

\section{Imaging results}

- General information on imaging examination

Among the 36 patients with COVID-19, during hospitalization, 5 patients underwent two chest CT scans, 10 patients underwent three CT scans, 13 patients underwent four CT scans, 6 patients underwent five CT scans, 1 patient underwent six CT scans, and 1 patient underwent seven CT scans. After discharge from the hospital, 16 patients underwent one follow-up chest CT scan, 9 patients underwent two follow-up CT 
scans, 4 patients underwent three follow-up CT scans, 3 patients underwent four follow-up CT scans, and 4 patients did not undergo follow-up CT.

- Imaging findings of the first appearance of "fibrosis-like" strips

"Fibrosis-like" strips on chest CT in 36 patients were all located in the subpleural region of the bilateral lungs. Twenty-nine patients (80.6\%) had "fibrosis-like" strips on the first chest CT scan, and 7 patients (19.4\%) showed "fibrosis-like" strips on the second CT scan; among them, 6 patients initially showed consolidation and ground-glass opacities in the bilateral lungs on the first chest CT scan, which evolved into "fibrosis-like" strips on the second CT scan, and, 1 patient initially showed patchy and ground-glass nodules in the bilateral lungs on the first chest CT scan, which also evolved into "fibrosis-like" strips on the second CT scan.

- Dynamic evolution of "fibrosis-like" strips

During hospitalization, "fibrosis-like" strips in 18 patients demonstrated a trend of gradually decreasing in number; "fibrosis-like" strips in 15 patients demonstrated a trend of first increasing in number, and then decreasing in number; and "fibrosis-like" strips in 3 patients demonstrated a trend of first decreasing in number, followed by an increase in number and then a decreasing in number. In addition, "fibrosis-like" strips containing bronchium gradually evolved into solid "fibrosis-like" strips in 15 patients. After discharge from the hospital, follow-up chest CT showed that "fibrosis-like" strips in 15 patients completely disappeared in the bilateral lungs, with a median absorption time of 37 days (range 19-124 days); in addition, "fibrosis-like" strips completely disappeared in the unilateral lung in 8 patients, with a median absorption time of 36 days (range 24-59 days); and finally, 13 patients showed a significant reduction in "fibrosis-like" strips in the bilateral lungs.

\section{Discussion}

Chest CT plays an important role in the diagnostic and prognostic assessment of COVID-19 pneumonia. The CT findings of COVID-19 pneumonia are complex and, include ground-glass nodules or ground-glass opacities, lung consolidation, thickening of the interlobular septum, "fibrosis-like" strips, air bronchogram signs, and thickening of blood vessels. Among these findings, "fibrosis-like" strips are found in some COVID-19 patients in both lungs on the first chest CT examination. At present, the exact imaging and pathological mechanism of "fibrosis-like" strips are still unclear. Many radiological experts in China believe that this finding is indicative of pulmonary fibrosis ${ }^{[2]}$. However, owing to the lack of systematic imaging studies and adequate pathological evidence, this conclusion is still controversial. Clinically, the prognosis of pulmonary fibrosis is poor, and the identification of pulmonary fibrosis would help clinicians determine whether the patient needs anti-fibrosis treatment. Therefore, the qualitative diagnosis of this sign has critical clinical significance. Lung biopsy is the gold standard for the diagnosis of pulmonary fibrosis. However, as an invasive procedure, lung biopsy not only increases the risk to patients, but also 
increases the risk of infection by medical staff. Therefore, it is difficult to obtain pathological specimens through lung biopsy in clinical practice.

Similar to COVID-19 pneumonia, SARS-CoV pneumonia is often combined with pulmonary fibrosis. It has been reported that the incidence of pulmonary fibrosis in SARS-CoV is as high as $25.9-62 \%{ }^{[3-4]}$. An autopsy study ${ }^{[5]}$ regarding SARS-related deaths demonstrated that pulmonary fibrosis appeared as early as 38 days after infection with the SARS virus. Wang et al. ${ }^{[6]}$ also found that strip-shaped fibrosis in patients with SARS first appeared 30-40 days after admission by follow-up CT. Lu et al. ${ }^{[7]}$ performed a CT follow-up study and observed that in 10 SARS patients with varying degrees of pulmonary fibrosis, the fibrosis was absorbed within 6 months, and pulmonary fibrosis in two patients lasted for 6 months and 12 months. Chen et al. ${ }^{[8]}$ also reported that $17.1 \%(19 / 111)$ of SARS patients still had residual pulmonary fibrosis 18 months after discharge. These studies suggest that pulmonary fibrosis in SARS patients has characteristics of late appearance and slow absorption.

A total of 36 COVID-19 patients with "fibrosis-like" strips were included in the present study. The initial chest CT scan on admission showed "fibrosis-like" strips in both lungs (Fig. 1a, 1e, 1i, 1m, 2a, 2b, 2e, 2f) in the majority of patients $(29 / 36,80.6 \%)$, with an average appearance time of 2 days (1-3 days). Seven patients $(7 / 36,19.4 \%)$ had "fibrosis-like" strips in both lungs on the second CT scan, with an average appearance time of 10 days (4-17 days). The average appearance time of "fibrosis-like" strips for all 36 patients was 2.7 days (1-17 days). All patients had different degrees of absorption of "fibrosis-like" strips after active intervention, and $41.7 \%$ of the patients showed complete absorption of "fibrosis-like" strips in the bilateral lungs (median 37 days, range 19-124 days). A total of $22.2 \%$ of the patients showed complete absorption of "fibrosis-like" strips in the unilateral lung (median 36 days, range 24-59 days). Additionally, $36.1 \%$ of the patients, showed a significant reduction of "fibrosis-like" strips in the bilateral lungs. Compared with previously published data regarding SARS-related pulmonary fibrosis ${ }^{[3-4,5-6,8]}$, "fibrosis-like" strips in COVID-19 pneumonia seem to appear earlier and be absorbed faster. Therefore, based on the imaging manifestations and outcome characteristics of "fibrosis-like" strips in the patients in the present study, we conclude that "fibrosis-like" strips in COVID-19 patients may not be pulmonary fibrosis.

On the first CT scan performed on day 1 at hospital in patients 6, "fibrosis-like" strips appeared in the bilateral lungs, and bronchia contained in the strips were observed (Fig. 2i). Moreover, the following three CT re-examinations (Fig. 2j-2l) showed that the number of strips with bronchia gradually decreased. Additionally, we used the bronchium contained in the "fibrosis-like" strip as the targeted bronchus for curved reformatting (Fig. 2m-2p), and observed a small nodular high-density shadow in the bronchium. Four consecutive CT scans (Fig. $2 \mathrm{~m}-2 \mathrm{p}$ ) showed that small nodular high-density shadows not only gradually decreased in number, but also changed in shape and position. Therefore, we speculate that the small nodular high-density shadows in the bronchium may be mucus embolus, while the strips may be pulmonary tissue atelectasis caused by the mucus embolus of the bronchium. The changes in the number, shape, and location of the small nodular high-density shadows in the bronchium suggest that 
they may indicate the process of mucus emboli being discharged from bronchia. At present, several pathological studies on COVID-19 pneumonia have also shown that mucus-like materials adhere to the walls of bronchia and/or mucus emboli in bronchia ${ }^{[9-13]}$.

Fig. 3a-v shows the evolution process of the "fibrosis-like" strips in another case of COVID-19 pneumonia, and the spatial relationships between the bronchia and strips were displayed visually on curved reformatted images (Fig. 3s-3v). On the first CT examination of the patient, bronchia contained in the "fibrosis-like" strips were observed in the bilateral lungs (Fig. 3a, 3g, 3m), which was considered inflammation in the lower lobes of both lungs with localized pulmonary atelectasis; the third CT scan showed decreased consolidation but increased strips in the bilateral lungs (Fig. 3c, 3i, 3o), suggesting reduced inflammation, but progressed pulmonary atelectasis. Subsequently, the fourth and fifth CT scans showed that the number of "fibrosis-like" strips containing bronchia (Fig. 3d, 3j, 3p) gradually decreased or even evolved into solid "fibrosis-like" strip (Fig. 3e, 3k, 3q), which may be associated with pulmonary atelectasis tissues gradually returning to normal when the inflammation was gradually absorbed after treatment. Therefore, the bronchia contained in the pulmonary atelectasis tissues also became gradually smooth and returned to normal; thus, the bronchia in the "fibrosis-like" strips gradually became invisible. The "fibrosis-like" strips were completely absorbed 43 days after onset (Fig. 3f, 3l, 3r). This typical case clearly demonstrates the evolution of the spatial relationships between "fibrosis-like" strips and bronchia over time, indicating that "fibrosis-like" strips are pulmonary segmental atelectasis rather than pulmonary fibrosis.

Although pulmonary fibrosis was observed in COVID-19 patients in several autopsy studies ${ }^{[9,11,12]}$, most of these death cases were related to severe and critical pneumonia, and they were associated with at least one underlying disease; therefore, the occurrence of pulmonary fibrosis may be associated with severe lung injury in these severe and critical cases.

For patients with mild and common COVID-19 pneumonia, the lung injury is relatively mild; in addition, the majority of "fibrosis-like" strips contain bronchia, and most of these lesions can be absorbed in a short period, which does not correspond to the imaging characteristics of pulmonary fibrosis.

In conclusion, "fibrosis-like" strips on chest CT scans in COVID-19 patients have the characteristics of early appearance and rapid morphological changes as well as rapid absorption. In addition, bronchia contained in "fibrosis-like" strips were observed in the majority of patients (88.9\%) in this study. Therefore, we speculate that "fibrosis-like" strips may not be pulmonary fibrosis, but a sign of pulmonary segmental atelectasis. However, the number of patients presenting with this typical evolution was relatively small, and all of the included patients in this study had mild or common COVID-1. The evidence is still insufficient, and further imaging-pathological correlation studies are needed to confirm our results.

Despite its limitations, this study certainly offers valuable insights into the potential pathogenesis of "fibrosis-like" strips in COVID-19 patients. 


\section{Declarations}

"The chest computed tomography images of the seven cases displaying "fibrosis-like" strips in both lungs on the consecutive CT scans are available in the figshare repository https://doi.org/10.6084/m9.figshare.13011962

\section{References}

[1] Coronavirus disease (COVID-19) Situation dashboard, WHO, 6:18pm CEST, 22 July 2020.

[2] Radiological Diagnosis of New Coronavirus Infected Pneumonitis: Expert Recommendation from the Chinese Society of Radiology (First edition), Chin J Radiol, 2020, 54: Epub ahead of print. DOI: 10.3760/cma.j.issn.1005-1201.2020.0001

[3] Antonio GE, Wong KT, Hui DS, et al. Thin-section CT in patients with severe acute respiratory syndrome following hospital discharge: preliminary experience. Radiology. 2003; 228(3):810-815. doi:10.1148/radiol.2283030726.

[4]Liu SL, LI KC, Wang ZQ, et al. Imaging Analysis of Thoracic Complications in Severe Acute Respiratory Syndrome [J]. Journal of Clinical Radiology, 2003, 22(8):657-659. DOI:10.3969/j.issn.10019324.2003.08.010.

[5]Wang DW, Li N, Zhao JM, et al. Pathology changes and characteristics in patients died at different times of critical SARS [J]. Medical Journal of Chinese People's Liberation Army, 2003, 28(8):692-696. DOI: 10.3321/j.issn:0577-7402.2003.08.012.

[6] Wang W, Ma DQ, Zhao DW, et al. Apearances and dynamic changes in severe acute respiratory syndrome [J] .Chinese Journal of Radiology, 2003, 37(8):686-689. DOI:10.3760/j.issn:10051201.2003.08.005.

[7] Lu PX, Yang GL, Yu WY, et al. Imaging follow-up of SARS patients complicated with pulmonary fibrosis [J]. Chinese Journal of Medical Imaging Technology, 2004, 20(12):1901-1903.DOI:10.3321/j.issn:10033289.2004.12.031.

[8] Chen JH, Ma DQ, He W, et al. Follow-up study of chest CT manifestations of patients with severe acute respiratory syndrome [J]. Chinese Journal of Radiology, 2006,40(11):1161-1165.DOI:10.3760/j.issn:10051201.2006.11.011.

[9] Yao XH, Li TY, He ZC, et al. A pathological report of three COVID-19 cases by minimal invasive autopsies. Chinese Journal of Pathology. 2020 May 8; 49(5):411-417.

DOI: 10.3760/cma.j.cn112151-20200312-00193. PMID: 32172546. 
[10] Wu JH, Li X, Huang B, et al. Pathological changes of fatal coronavirus disease 2019 (COVID-19) in the lungs: report of 10 cases by postmortem needle autopsy. Chinese Journal of Pathology. 2020 Jun 8; 49(6):568-575. DOI: 10.3760/cma.j.cn112151-20200405-00291. PMID: 32486534.

[11] Liu Q, Wang RS, Qu GQ, et al. General anatomy report of novel coronavirus pneumonia death corpse [J]. Journal of Forensic Medicine, 2020, 01:21-23.

[12] Luo W, Yu H, Gao J, et al. Clinical pathology of critical patient with novel coronavirus pneumonia (COVID-19). Preprints 2020, 2020020407.

[13] Novel coronavirus pneumonia diagnosis and treatment plan (Trial Seventh Edition), national health and Health Committee Office.2020-03-03.

Sun, Q. et al. Computed tomography images used to investigate whether "fibrosis-like" strips on chest CT in patients with COVID-19 pneumonia true

fibrosis. figshare https://doi.org/10.6084/m9.figshare.13011962 (2020).

\section{Figures}



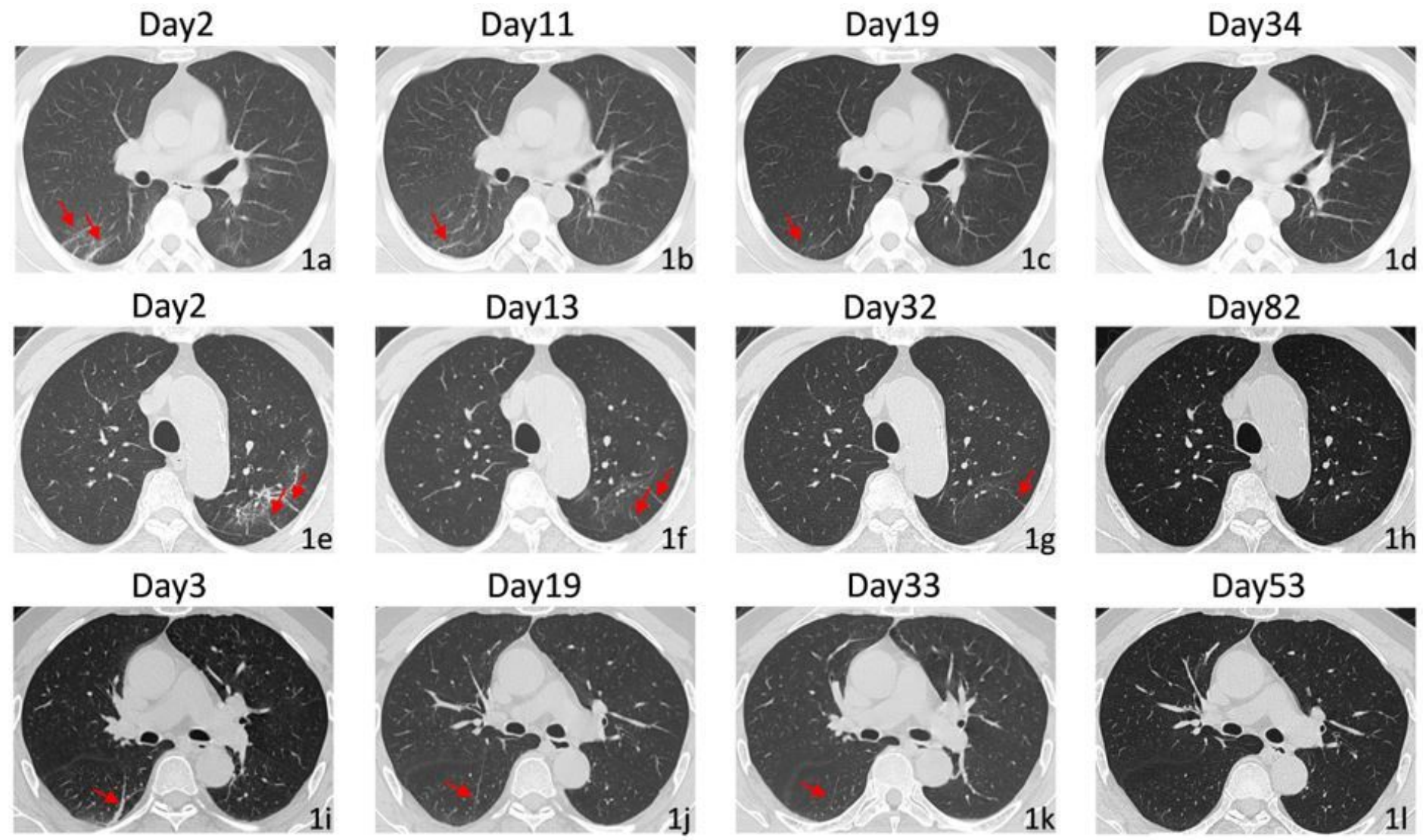

Day1
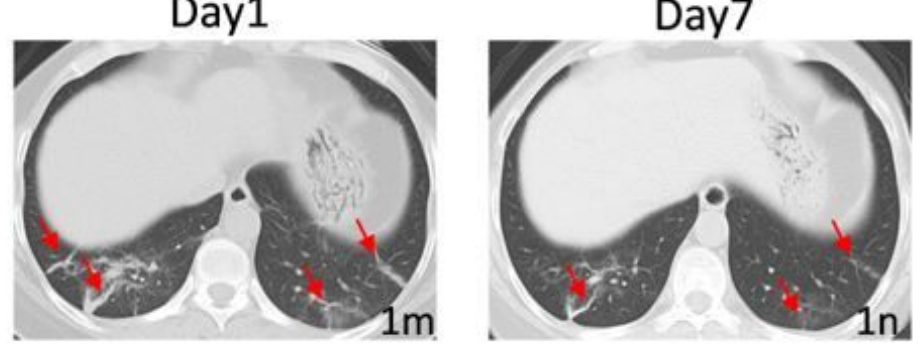

\section{Day16}

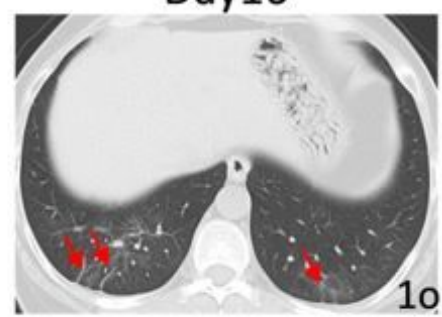

\section{Day51}

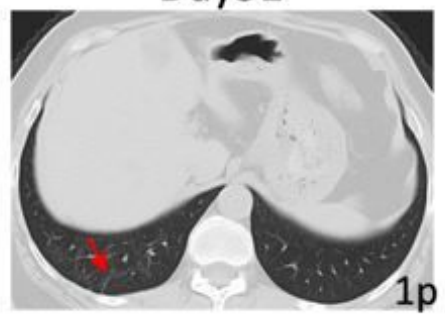

\section{Figure 1}

a-d Images from case 1. (Fig 1a) First CT performed on day 2 shows "fibrosis-like" strips in subpleural region of right lung; (Fig 1b) Second CT performed on day 11 shows significantly decreased "fibrosis-like" strips in subpleural region of right lung; (Fig 1C) Third CT performed on day 19 shows decreased "fibrosislike" strips in subpleural region of right lung; (Fig 1d) Fourth CT performed on day 34 shows "fibrosis-like" strips disappear in subpleural region of right lung. Fig1e-h Images from case 2. (Fig 1e) First CT performed on day 2 shows ground-glass opacities and "fibrosis-like" strips in subpleural region of left lung; (Fig 1f) Second CT performed on day 13 shows significantly decreased ground-glass opacities and "fibrosis-like" strips in subpleural region of left lung; (Fig 1g) Third CT performed on day 32 shows the further decreased "fibrosis-like" strips in subpleural region of left lung; (Fig 1h) Fourth CT performed on day 82 shows "fibrosis-like" strips disappear. Fig1i-I Images from case 3. (Fig 1i) First CT performed on day 3 shows "fibrosis-like" strip in subpleural region of right lung; (Fig 1j) Second CT performed on day 19 shows decreased "fibrosis-like" strip in subpleural region of right lung; (Fig 1k) Third CT performed on day 33 shows "fibrosis-like" strip in subpleural region of right lung become almost invisible; (Fig 1I) Fourth CT 
performed on day 53 shows "fibrosis-like" strip in subpleural region of right lung disappear. Fig1m-p Images from case 4. (Fig 1m) First CT performed on day 1 shows "fibrosis-like" strips containing bronchium in bilateral inferior lobes; (Fig 1n) Second CT performed on day 7 shows the size of "fibrosislike" strips containing bronchium decreased in bilateral inferior lobes; (Fig 10) Third CT performed on day 16 shows "fibrosis-like" strips containing bronchium evolved into solid "fibrosis-like" in bilateral inferior lobes; (Fig 1p) Fourth CT performed on day 51 shows "fibrosis-like" strips are completely absorbed in subpleural region of left lung, and "fibrosis-like" strips became almost invisible in subpleural region of right lung.

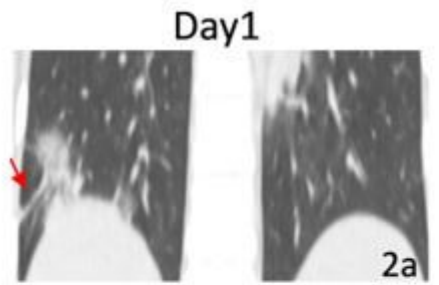

Day1

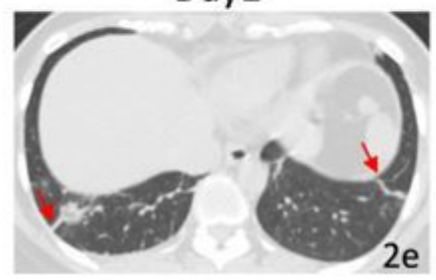

Day1

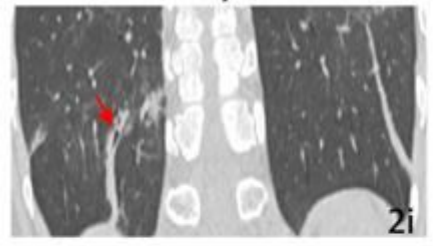

Day1

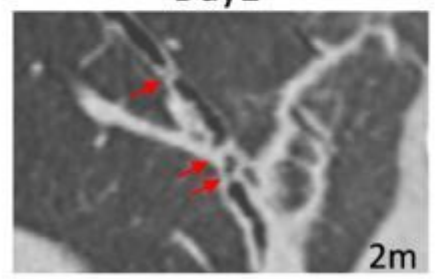

Day1

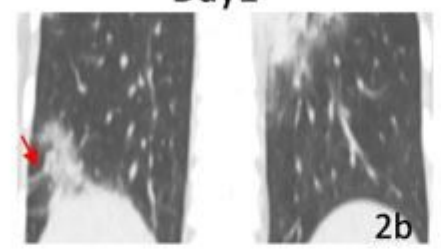

Day1

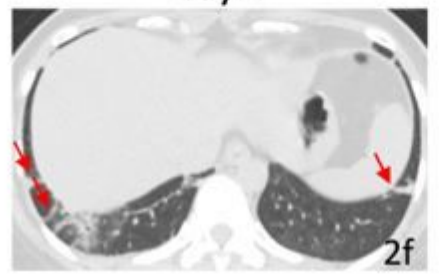

Day7

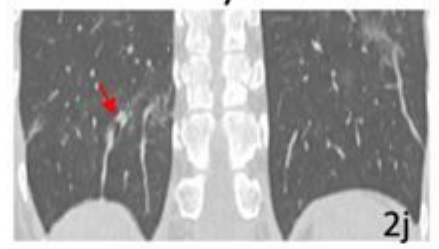

Day7

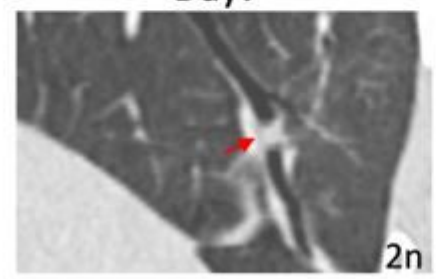

Day7

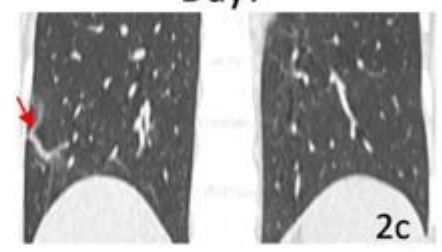

Day7

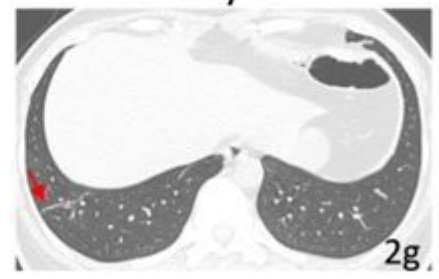

Day16

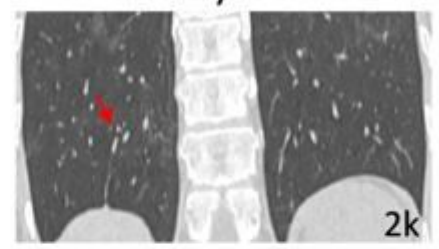

Day16

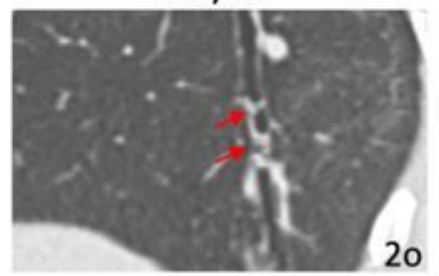

Day13

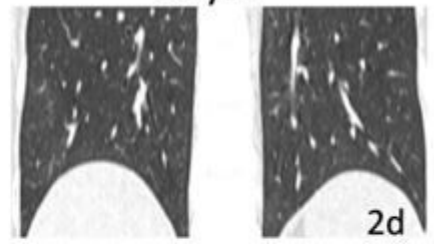

Day13

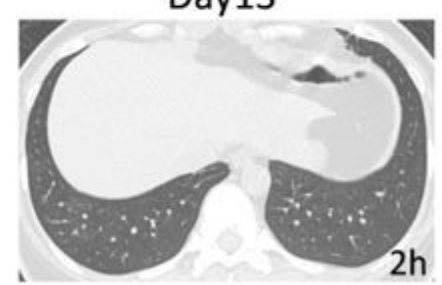

Day48

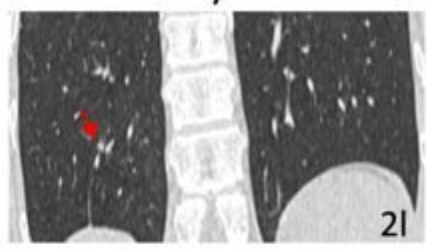

Day48

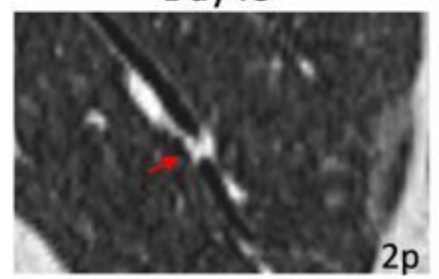

\section{Figure 2}

a-h Images from case 5. (Fig 2a-b囚2e-f) First CT performed on day 1 shows "fibrosis-like" strips and ground-glass opacities in bilateral inferior lobes; (Fig 2c $\unrhd$ ) Second CT performed on day 7 shows significantly decreased "fibrosis-like" strips in subpleural region of right lung, besides, "fibrosis-like" strips in subpleural region of left lung disappear; (Fig 2d $\mathrm{d}$ h) Third CT performed on day 13 shows "fibrosis-like" strips in subpleural region of right lung disappear. Fig2i-p Images from case 6, Fig $2 m-2 p$ are the curved reformatted images of the target bronchium (red arrow) contained in the "fibrosis-like" strips which are shown in Fig2i-2I. (Fig 2i) First CT performed on day 1 shows "fibrosis-like" strips containing bronchium in subpleural region of right lung and solid "fibrosis-like" strip in subpleural region of left lung, (Fig $2 \mathrm{~m}$ ) The 
curved reformatted image shows multiple nodules of high density are observed in the targeted bronchium; (Fig 2j) Second CT performed on day 7 shows the size of "fibrosis-like" strips decreased in bilateral inferior lobes, (Fig 2n) The curved reformatted image shows the morphology and location of nodules changed in the targeted bronchium; (Fig 2k) Third CT performed on day 16 shows the further decreased "fibrosis-like" strips in subpleural region of right lung, "fibrosis-like" strips in subpleural region of left lung disappear, (Fig 20)The curved reformatted image shows the location of the nodule changed again in the targeted bronchium; (Fig 2l) Fourth CT performed on day 48 shows the size of "fibrosis-like" strips decreased a little, (Fig $2 p$ ) The curved reformatted image shows the nodules are smaller in the target bronchium than before.
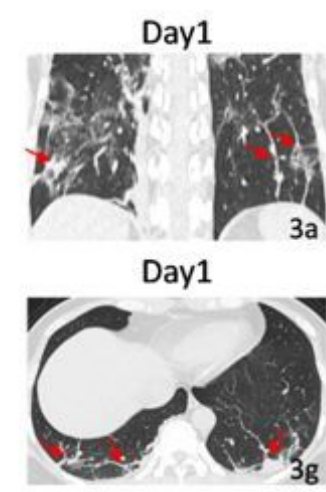

Day1

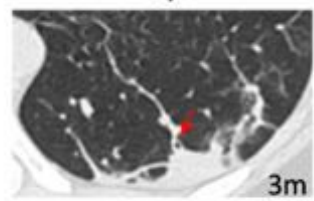

Day1

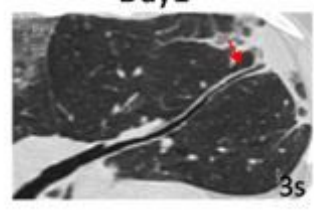

Day6

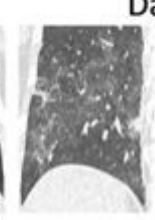

Day6

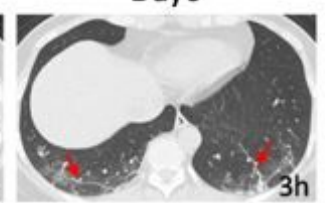

Day6

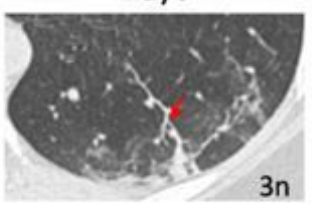

$3 n$

Day6

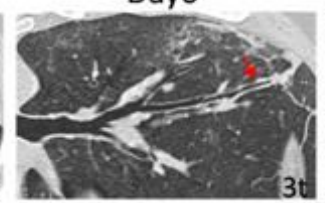

Day19

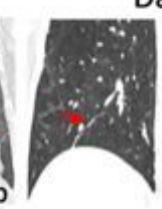

Day19

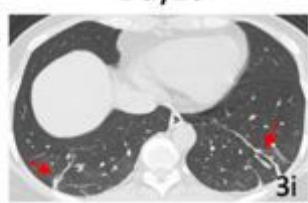

Day19

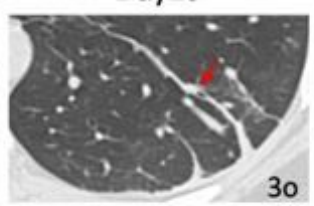

Day19

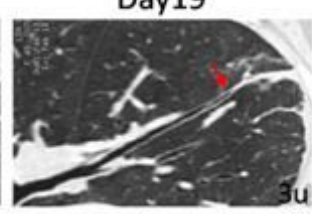

Day24

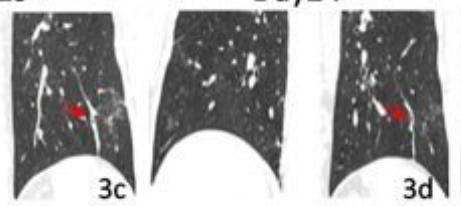

Day24

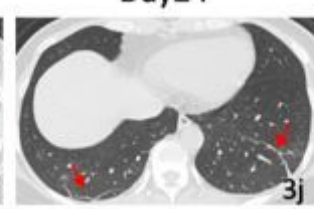

Day24

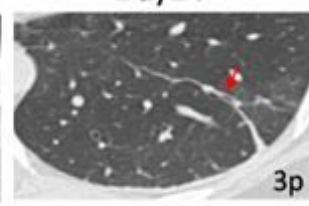

$3 p$

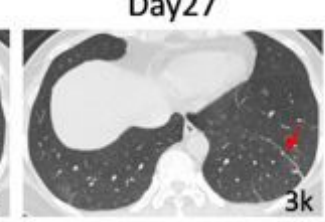

Day27

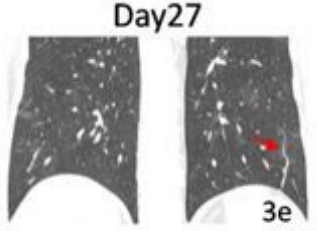

Day27

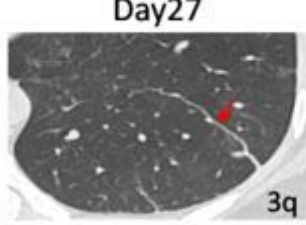

Day24

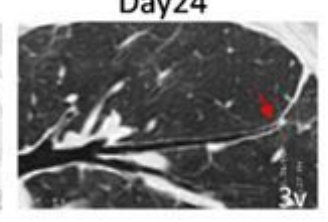

Day43

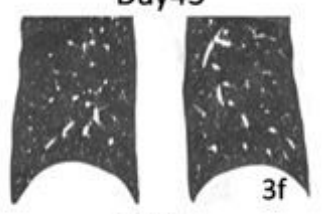

Day43

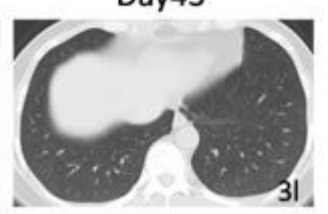

Day43

\section{Figure 3}

a-v Images from case 7. Fig3s-v shows the curved reformatted images of the targeted bronchium (red arrow) contained in the "fibrosis-like" strip (Fig 3m-p). (Fig 3a, 3g, 3m, 3s) First CT performed on day 1 shows "fibrosis-like" strips in bilateral subpleural regions and consolidation in subpleural region of left lung; (Fig 3b, 3h, 3n, 3t) Second CT performed on day 6 shows shrunken "fibrosis-like" strips in bilateral subpleural regions, consolidation in subpleural region of left lung almost disappear; (Fig3c, 3i, 3o, 3u) Third CT performed on day 19 shows increased "fibrosis-like" strips in bilateral subpleural regions, some "fibrosis-like" strips containing bronchium;(Fig 3d, 3j, 3p, 3v) Fourth CT performed on day 24 shows decreased "fibrosis-like" strips in bilateral subpleural regions;(Fig 3e, 3k, 3q) Fifth CT performed on day 27 shows "fibrosis-like" strip containing bronchium evolved into solid "fibrosis-like" strip in subpleural region of left lung, the "fibrosis-like" strips become almost invisible in subpleural region of right lung; (Fig 3f囚3I 3r) Sixth CT performed on day 43 shows "fibrosis-like" strips disappear in bilateral subpleural regions. 